\title{
Dark Matter, a Direct Detection
}

\section{Stéphane Le Corre}

Ecole Polytechnique Fédérale de Lausanne, Lausanne, $\mathrm{CH}$, Switzerland

Email: stephane.lecorre@epfl.ch

How to cite this paper: Le Corre, S. (2017) Dark Matter, a Direct Detection. Open Access Library Journal, 4: e4219. https://doi.org/10.4236/oalib.1104219

Received: November 29, 2017

Accepted: December 22, 2017

Published: December 25, 2017

Copyright (C) 2017 by author and Open Access Library Inc.

This work is licensed under the Creative Commons Attribution International License (CC BY 4.0).

http://creativecommons.org/licenses/by/4.0/

\begin{abstract}
In a previous paper, we demonstrated that the linearized general relativity could explain dark matter (the rotation speed of galaxies, the rotation speed of dwarf satellite galaxies, the movement in a plane of dwarf satellite galaxies, the decreasing quantity of dark matter with the distance to the center of galaxies' cluster, the expected quantity of dark matter inside galaxies and the expected experimental values of parameters $\Omega_{\mathrm{dm}}$ of dark matter measured in CMB). It leads, compared with Newtonian gravitation, to taking in account the second component (gravitational field) of the gravitation (imposed by general relativity) without changing the gravity field (also known as gravitomagnetism). In this explanation, dark matter would be a uniform gravitational field that embeds some very large areas of the universe generated by the clusters. In this article we are going to see that this specific gravitational field, despite its weakness, could be soon detectable, allowing testing this explanation of dark matter. It should generate a slight discrepancy in the expected measure of the Lense-Thirring effect of the Earth. In this theoretical frame, the Lense-Thirring effect of the "dark matter" would be a value between around 0.3 milliarcsecond/year and 0.6 milliarcsecond/year in the best case. In the LAGEOS or Gravity Probe B experiments, there was not enough precision (around 0.3\% for the expected 6606 mas $\cdot \mathrm{y}^{-1}$ geodetic and around 19\% for the expected 39 mas $\cdot \mathrm{y}^{-1}$ frame-dragging precessions). In the GINGER experiment, there could be enough one; the expected accuracy would be around $1 \%$. If this discrepancy was verified, it would be the first direct measure of the dark matter.
\end{abstract}

\section{Subject Areas}

Modern Physics, Theoretical Physics

\section{Keywords}

Dark Matter, Galaxies: Formation, Galaxies: Evolution, Gravitation 


\section{Introduction}

One of the most important mysteries of astrophysics is the problem of dark matter. This latter component represents at least five times the quantity of the ordinary mass. And until now, this term cannot be explained. We therefore find that our theories operate in a highly coherent and precise manner both on our scale and at large astrophysical scales. But as far as large scales are concerned, this coherence and precision is only possible on the condition of making the hypothesis of the existence of this new term of dark matter. A way to solve this problem is to propose new theories (MOND theories for example), but we can note that the term of dark matter, even if it is an ad hoc term, doesn't generate any contradiction inside our current theories. In fact, we could even pretend that this term demonstrates the extraordinary consistency of our current theories because of the multiplicity of the ways to deduce the quantity of this term leading to its more and more coherent and precise measure. Another way is to propose that this term represents a new exotic matter. This explanation is more shared and certainly more studied. But until now, no new matter has been directly detected, despite more and more experiences. A third way is to propose an explanation in the frame of current theories. That's the purpose of the present work. The term of dark matter will be explained by a physical phenomenon of general relativity that is generally neglected.

General relativity implies the existence of two gravitational components. In addition to the gravity field, there is a gravitational field (together giving what is called the gravitomagnetism) just like the magnetic field in electromagnetism. The new gravitational field can be measured by its precession effect, known as Lense-Thirring effect. Several experiments have validated this effect for the Earth gravitational field, NASA's LAGEOS satellites or Gravity Probe B [1] with an accuracy of around $19 \%$. Some new experiments will try to obtain a higher accuracy, for example GINGER [2] with an expected accuracy of around $1 \%$.

In [3], a solution is proposed to explain the dark matter, compliant with general relativity. This explanation leads to the assumption that we are embedded in a relatively uniform gravitational field generated by larger structures than galaxies (likely the clusters). Just like the Earth gravitational field can be measured, this hypothetical embedding gravitational field could be measured by its precession effect (Lense-Thirring effect). Such a measure will be a direct measure of the "dark matter". We are going to calculate the magnitude of this measure. This value is consistent with its non-detection until now. But it seems accessible to the next generation of experiments. In the most advantageous case, the accuracy of $1 \%$ (as expected in GINGER experiment) could be enough to detect it.

Before recalling the theoretical idealization used and some of the results of [3], it is remarkable to note that in this theoretical solution, dark matter is not some matter but a part of the second component of the gravitation term, term that exists only in the general relativity. It means that this explanation is available only in the frame of the general relativity. This remark also implies that the detection 
of particles of dark matter would be doomed to failure (it would be equivalent to search for particles of magnetic field). With general relativity, the ordinary matter would be sufficient to generate the gravitational field to explain the dark matter term (just like charged particles generate magnetic field). Dark matter would be then another proof of the power of this theoretical frame (of the same importance as curvature of light trajectories, Lense-Thirring effect or gravitational waves).

\section{Dark Matter Explained by General Relativity}

\subsection{From General Relativity to Linearized General Relativity}

From general relativity, one deduces the linearized general relativity in the approximation of a quasi-flat Minkowski space ( $\left.g^{\mu v}=\eta^{\mu v}+h^{\mu \nu} ;\left|h^{\mu v}\right| \ll 1\right)$. With the following Lorentz gauge, it gives the following field equations as in [4] (with $\left.\square=\frac{1}{c^{2}} \frac{\partial^{2}}{\partial t^{2}}-\Delta\right)$ :

$$
\partial_{\mu} \bar{h}^{\mu v}=0 ; \quad \square \bar{h}^{\mu v}=-2 \frac{8 \pi G}{c^{4}} T^{\mu v}
$$

With:

$$
\begin{aligned}
& \bar{h}^{\mu v}=h^{\mu \nu}-\frac{1}{2} \eta^{\mu v} h ; h \equiv h_{\sigma}^{\sigma} ; h_{v}^{\mu}=\eta^{\mu \sigma} h_{\sigma v} ; \\
& \bar{h}=-h
\end{aligned}
$$

The general solution of these equations is:

$$
\bar{h}^{\mu v}(c t, \boldsymbol{x})=-\frac{4 G}{c^{4}} \int \frac{T^{\mu v}(c t-|\boldsymbol{x}-\boldsymbol{y}|, \boldsymbol{y})}{|\boldsymbol{x}-\boldsymbol{y}|} \mathrm{d}^{3} \boldsymbol{y}
$$

In the approximation of a source with low speed, one has:

$$
T^{00}=\rho c^{2} ; T^{0 i}=c \rho u^{i} ; T^{i j}=\rho u^{i} u^{j}
$$

And for a stationary solution, one has:

$$
\bar{h}^{\mu \nu}(\boldsymbol{x})=-\frac{4 G}{c^{4}} \int \frac{T^{\mu \nu}(\boldsymbol{y})}{|\boldsymbol{x}-\boldsymbol{y}|} \mathrm{d}^{3} \boldsymbol{y}
$$

At this step, by proximity with electromagnetism, one traditionally defines a scalar potential $\varphi$ and a vector potential $H^{i}$. There are in the literature several definitions as in [5] for the vector potential $H^{i}$. In our study, we are going to define:

$$
\bar{h}^{00}=\frac{4 \varphi}{c^{2}} ; \bar{h}^{0 i}=\frac{4 H^{i}}{c} ; \bar{h}^{i j}=0
$$

With gravitational scalar potential $\varphi$ and gravitational vector potential $H^{i}$ :

$$
\begin{aligned}
& \varphi(\boldsymbol{x}) \equiv-G \int \frac{\rho(\boldsymbol{y})}{|\boldsymbol{x}-\boldsymbol{y}|} \mathrm{d}^{3} \boldsymbol{y} \\
& H^{i}(\boldsymbol{x}) \equiv-\frac{G}{c^{2}} \int \frac{\rho(\boldsymbol{y}) u^{i}(\boldsymbol{y})}{|\boldsymbol{x}-\boldsymbol{y}|} \mathrm{d}^{3} \boldsymbol{y}=-K^{-1} \int \frac{\rho(\boldsymbol{y}) u^{i}(\boldsymbol{y})}{|\boldsymbol{x}-\boldsymbol{y}|} \mathrm{d}^{3} \boldsymbol{y}
\end{aligned}
$$


With $K$ a new constant defined by:

$$
G K=c^{2}
$$

This definition gives $K^{-1} \sim 7.4 \times 10^{-28}$ very small compare to $G$.

The field equations (1) can be then written (Poisson equations):

$$
\Delta \varphi=4 \pi G \rho ; \Delta H^{i}=\frac{4 \pi G}{c^{2}} \rho u^{i}=4 \pi K^{-1} \rho u^{i}
$$

With the following definitions of $\boldsymbol{g}$ (gravity field) and $\boldsymbol{k}$ (gravitational field), those relations can be obtained from the following equations (also called gravitomagnetism):

$$
\begin{aligned}
& \boldsymbol{g}=-\operatorname{grad} \varphi ; \boldsymbol{k}=\operatorname{rot} \boldsymbol{H} \\
& \operatorname{rot} \boldsymbol{g}=0 ; \operatorname{div} \boldsymbol{k}=0 ; \\
& \operatorname{div} \boldsymbol{g}=-4 \pi G \rho ; \operatorname{rot} \boldsymbol{k}=-4 \pi K^{-1} \boldsymbol{j}_{p}
\end{aligned}
$$

With the Equations (2), one has:

$$
h^{00}=h^{11}=h^{22}=h^{33}=\frac{2 \varphi}{c^{2}} ; h^{0 i}=\frac{4 H^{i}}{c} ; h^{i j}=0
$$

The equations of geodesics in the linear approximation give:

$$
\frac{\mathrm{d}^{2} x^{i}}{\mathrm{~d} t^{2}} \sim-\frac{1}{2} c^{2} \delta^{i j} \partial_{j} h_{00}-c \delta^{i k}\left(\partial_{k} h_{0 j}-\partial_{j} h_{0 k}\right) v^{j}
$$

It then leads to the movement equations:

$$
\frac{\mathrm{d}^{2} \boldsymbol{x}}{\mathrm{d} t^{2}} \sim-\operatorname{grad} \varphi+4 \boldsymbol{v} \wedge(\boldsymbol{r o t} \boldsymbol{H})=\boldsymbol{g}+4 \boldsymbol{v} \wedge \boldsymbol{k}
$$

Remark: Afterwards, we will use relations defined with the parameterized post-Newtonian formalism (PPN). All previous relations can be retrieved starting with the PPN formalism. From [6] one has:

$$
g_{0 i}=-\frac{1}{2}\left(4 \gamma+4+\alpha_{1}\right) V_{i} ; V_{i}(\boldsymbol{x})=\frac{G}{c^{2}} \int \frac{\rho(\boldsymbol{y}) u_{i}(\boldsymbol{y})}{|\boldsymbol{x}-\boldsymbol{y}|} \mathrm{d}^{3} \boldsymbol{y}
$$

The gravitomagnetic field and its acceleration contribution are:

$$
\boldsymbol{B}_{g}=\nabla \wedge\left(g_{0 i} e^{i}\right) ; \boldsymbol{a}_{g}=\boldsymbol{v} \wedge \boldsymbol{B}_{g}
$$

And in the case of general relativity (that is our case):

$$
\gamma=1 ; \alpha_{1}=0
$$

It then gives:

$$
g_{0 i}=-4 V_{i} ; \quad \boldsymbol{B}_{g}=\nabla \wedge\left(-4 V_{i} \boldsymbol{e}^{i}\right)
$$

And with our definition:

$$
H_{i}=-\delta_{i j} H^{j}=\frac{G}{c^{2}} \int \frac{\rho(\boldsymbol{y}) \delta_{i j} u^{j}(\boldsymbol{y})}{|\boldsymbol{x}-\boldsymbol{y}|} \mathrm{d}^{3} \boldsymbol{y}=V_{i}(\boldsymbol{x})
$$

One then has:

$$
g_{0 i}=-4 H_{i} ; \quad \boldsymbol{B}_{g}=\nabla \wedge\left(-4 H_{i} \boldsymbol{e}^{i}\right)=\nabla \wedge\left(4 \delta_{i j} H^{j} \boldsymbol{e}^{i}\right)=4 \nabla \wedge \boldsymbol{H}
$$




$$
\boldsymbol{B}_{g}=4 \operatorname{rot} \boldsymbol{H}
$$

With the following definition of gravitational field:

$$
\boldsymbol{k}=\frac{\boldsymbol{B}_{g}}{4}
$$

One then retrieves our previous relations:

$$
\boldsymbol{k}=\boldsymbol{r o t} \boldsymbol{H} ; \boldsymbol{a}_{g}=\boldsymbol{v} \wedge \boldsymbol{B}_{g}=4 \boldsymbol{v} \wedge k
$$

A last remark: The interest of our notation is that the field equations are strictly equivalent to Maxwell idealization (in particular the speed of the gravitational wave obtained from these equations is the light celerity). Only the movement equations are different with the factor " 4 ". But of course, all the results of our study could be obtained in the traditional notation of gravitomagnetism with the relation $\boldsymbol{k}=\frac{\boldsymbol{B}_{g}}{4}$.

\subsection{From Linearized General Relativity to Dark Matter}

The article [3] demonstrates that the dark matter can be explained by the gravitational field $\boldsymbol{k}$. It means that dark matter shouldn't be some matter but the effect of a gravitational field. This field is very similar to the magnetic field in electromagnetism. But, in the dynamic of a galaxy, this term is neglected. One of the main results of [3] is the expected values of this field being able to explain the curve of rotation of the galaxies, with $\boldsymbol{k}_{0}$ the gravitational field of "dark matter" (Table 1):

$$
10^{-16.62}<\left\|\boldsymbol{k}_{0}\right\|<10^{-16.3}
$$

As demonstrated in [3], these values justify that it can't be produced by the galaxy, in agreement with the fact that this term for galaxies can be neglected. But, these values should be able to be produced by the clusters, embedding then large areas of the Universe.

\section{Gravitational Field of Clusters (Dark Matter): A Way to Directly Measure Dark Matter}

Just like for the electromagnetism with the magnetic field, this gravitational field implies a phenomenon of precession. It is known as the Lense-Thirring effect. As this gravitational field of clusters should embed large area of the Universe, it should in particular embed the earth. So, instead of taking into account only the own gravitational field of the earth, we are also going to take into account the hypothetical embedding gravitational field that explains the dark matter. We are first going to recall what the equations in the general relativity are for the Lense-Thirring effect. And secondly, we will use it to test this solution by calculating the contribution to the precession effect generated by this gravitational field that explains the dark matter.

\subsection{Gravitational Field and Precession Effect}

The equations of the motion for the spin four-vector $S_{\mu}$ have been studied in several papers. In general relativity, it leads to a precession of $S_{\mu}$. It can be 
Table 1. Distance $r_{0}$ to the center of the galaxy where the internal gravitational field $\frac{K_{1}}{r^{2}}$ generated by the galaxy becomes equivalent to the external gravitational field $k_{0}$ generated by the galaxies' cluster.

\begin{tabular}{|c|c|c|c|c|}
\hline & $K_{1}$ & $k_{0}$ & $r_{0}\left[\frac{K_{1}}{r^{2}} \sim k_{0}\right]$ & $r_{0}[\mathrm{kpc}]$ \\
\hline NGC 5055 & $10^{24.6}$ & $10^{-16.62}$ & $10^{20.61}$ & 13 \\
\hline NGC 4258 & $10^{24.85}$ & $10^{-16.54}$ & $10^{20.695}$ & 16 \\
\hline NGC 5033 & $10^{24.76}$ & $10^{-16.54}$ & $10^{20.65}$ & 15 \\
\hline NGC 2841 & $10^{24.85}$ & $10^{-16.33}$ & $10^{20.59}$ & 13 \\
\hline NGC 3198 & $10^{24.9}$ & $10^{-16.55}$ & $10^{20.725}$ & 18 \\
\hline NGC 7331 & $10^{24.18}$ & $10^{-16.3}$ & $10^{20.24}$ & 6 \\
\hline NGC 2903 & $10^{24.71}$ & $10^{-16.3}$ & $10^{20.505}$ & 11 \\
\hline NGC 3031 & $10^{24.15}$ & $10^{-16.57}$ & $10^{20.36}$ & 8 \\
\hline NGC 2403 & $10^{24.59}$ & $10^{-16.39}$ & $10^{20.49}$ & 10 \\
\hline NGC 247 & $10^{24.3}$ & $10^{-16.3}$ & $10^{20.3}$ & 7 \\
\hline NGC 4236 & $10^{24}$ & $10^{-16.34}$ & $10^{20.17}$ & 5 \\
\hline NGC 4736 & $10^{24.54}$ & $10^{-16.3}$ & $10^{20.42}$ & 9 \\
\hline NGC 300 & $10^{24.27}$ & $10^{-16.31}$ & $10^{20.29}$ & 6 \\
\hline NGC 2259 & $10^{24.2}$ & $10^{-16.3}$ & $10^{20.25}$ & 6 \\
\hline NGC 3109 & $10^{24}$ & $10^{-16.58}$ & $10^{20.29}$ & 6 \\
\hline NGC 224 & $10^{24}$ & $10^{-16.5}$ & $10^{20.25}$ & 6 \\
\hline
\end{tabular}

deduced from the equations seen in paragraph 2.1. From [1], one can write the following equations:

$$
\dot{\boldsymbol{S}}=\left[\left(\gamma+\frac{\alpha}{2}\right) \frac{1}{c^{2}}(\operatorname{grad} \varphi \wedge \boldsymbol{v})+\frac{1}{4}(\gamma+\alpha) \operatorname{roth}\right] \wedge \boldsymbol{S}
$$

Which lead to define a geodetic vector field $\boldsymbol{\Omega}_{G}$ and a "gravito-magnetic" (frame-dragging) vector field $\boldsymbol{\Omega}_{L T}$ :

$$
\boldsymbol{\Omega}_{G}=\left(\gamma+\frac{\alpha}{2}\right) \frac{1}{c^{2}}(\operatorname{grad} \varphi \wedge \boldsymbol{v}) ; \boldsymbol{\Omega}_{L T}=\frac{1}{4}(\gamma+\alpha) \text { roth }
$$

These expressions use the PPN formalism. For general relativity, $\alpha=1$ and as seen before (16), $\gamma=1$, it leads to:

$$
\Omega_{G}=\frac{3}{2 c^{2}} \operatorname{grad} \varphi \wedge v ; \Omega_{L T}=\frac{1}{2} \operatorname{roth}
$$

In our notation (20):

$$
\boldsymbol{H}=\frac{\boldsymbol{h}}{4} ; \boldsymbol{k}=\boldsymbol{r o t} \boldsymbol{H}
$$

One then has

$$
\boldsymbol{\Omega}_{L T}=2 \boldsymbol{k}
$$




\subsection{Measure of the Dark Matter}

In our solution, around the Earth, $\boldsymbol{k}$ represents the addition of two terms, the own gravitational field of the earth $\boldsymbol{k}_{E}$ and the external uniform gravitational field of the clusters (the dark matter) $\boldsymbol{k}_{0}$ :

$$
\boldsymbol{k}=\boldsymbol{k}_{E}+\boldsymbol{k}_{0}
$$

In the same way, the Lense-Thirring effect $\Omega_{L T}$ is then composed of the own Earth gravitational field term $\Omega_{L T_{-} E}$ and of a new supplementary term of "dark matter" $\boldsymbol{\Omega}_{L T+D M}$

$$
\boldsymbol{\Omega}_{L T}=2 \boldsymbol{k}_{E}+2 \boldsymbol{k}_{0}=\boldsymbol{\Omega}_{L T_{-} E}+\boldsymbol{\Omega}_{L T_{-} D M}
$$

The term $\Omega_{L T_{-} E}$ is the traditional frame-dragging precession:

$$
\boldsymbol{H}_{E}=\frac{\boldsymbol{h}_{E}}{4}=\left(\frac{G}{2 c^{2} r^{3}}\right)(\boldsymbol{r} \wedge \boldsymbol{J}) ; \boldsymbol{\Omega}_{L T_{-} E}=\frac{G}{c^{2}}\left(\frac{\boldsymbol{J}}{r^{3}}-\frac{3 \boldsymbol{r}}{r^{5}}(\boldsymbol{r} \cdot \boldsymbol{J})\right)
$$

In the Gravity Probe B experiment, the expected value for the frame-dragging precession was:

$$
\left\|\boldsymbol{\Omega}_{L T_{-} G P B}\right\|=39 \text { milliarcsecond/year }
$$

Let's evaluate the order of magnitude of the external gravitational field (the dark matter) around the Earth. From [3], an average value is:

$$
\begin{aligned}
& \left\|\boldsymbol{\Omega}_{L T_{-} D M}\right\|=2\left\|\boldsymbol{k}_{0}\right\| \sim 2 \times 10^{-16.5} \mathrm{~s}^{-1} \\
& \left\|\boldsymbol{\Omega}_{L T_{-} D M}\right\|=0.4 \text { milliarcsecond/year }
\end{aligned}
$$

In fact, from the sample of galaxies studied in [3], one obtains the interval (22) for $k_{0}$. If we assume that these galaxies can be representative of our own galaxy, the expected discrepancy should be in the following interval:

$$
0.3<\left\|\Omega_{L T_{-} D M}\right\|<0.6 \text { (milliarcsecond/year) }
$$

\subsection{Discussion about the Expected Value of Lense-Thirring Effect}

$\left\|\boldsymbol{\Omega}_{L T_{-} D M}\right\|$ represents around $1 \%$ of $\left\|\boldsymbol{\Omega}_{L T_{-} G P B}\right\|$, (indeed 0.4 mas $\cdot \mathrm{y}^{-1} \sim 0.01 \times 39$ mas $\left.\cdot \mathrm{y}^{-1}\right)$. But until now, $\left\|\boldsymbol{\Omega}_{L T_{-} G P B}\right\|$ is only known with a precision $19 \%$ [1]. We need to have a better accuracy on this kind of experiments to hope to detect this discrepancy. GINGER experiment should have a precision of $1 \%$. It could be enough to begin to detect a discrepancy.

But there are several aspects of the experiment that can play a role in decreasing or increasing this discrepancy. The Sun is at around $8 \mathrm{kpc}$ from the galactic center. In [3] we have seen that at this distance the gravitational field of the galaxy could be of the same magnitude than $\boldsymbol{k}_{0}$ (column $r_{0}[\mathrm{kpc}]$ in Table 1). Therefore the expected values should be around 2 times greater than the previous calculated values of $\left\|\Omega_{L T+D M}\right\|$. But one also have to take into account the unknown direction of $\boldsymbol{k}_{0}$, implying that the magnitude of the effect could be reduced. The values previously calculated are obtained in the more advantageous case. 


\section{Conclusion}

In [3], general relativity explains the dark matter as the gravitational field of the clusters. This solution implies a Lense-Thirring effect detectable around the Earth with some experiments as Gravity Probe B. Compared to the expected value of the frame-dragging precession around the Earth $\left(\boldsymbol{\Omega}_{L T_{-} G P B}=39\right.$ milliarcsecond/year $)$, in this solution, the dark matter should generate a discrepancy in the following interval (in milliarcsecond/year) $0.3<\left\|\Omega_{L T_{-} D M}\right\|<0.6$ (for the most advantageous direction of $\boldsymbol{k}_{0}$ ). The previous experiments (LAGEOS or Gravity Probe B) didn't have sufficient accuracy (19\% for the measure of the frame-dragging precession in Gravity Probe B). The expected discrepancy needs at least a precision of $1 \%$. The next generation of experiments (as GINGER) will have such an accuracy. So, in the best case, a precision of $1 \%$ could begin to reveal a discrepancy in the measure of the expected precession effects. Without any detection, a higher accuracy would still be required to definitively declare that this solution is irrelevant. In particular if the direction of $\boldsymbol{k}_{0}$ is very disadvantageous. But if this discrepancy was measured, it will be the first direct measure of the dark matter.

\section{References}

[1] Adler, R.J. (2015) The Three-Fold Theoretical Basis of the Gravity Probe B Gyro Precession Calculation. https://doi.org/10.1088/0264-9381/32/22/224002

[2] Ruggiero, M.L. (2015) Sagnac Effect, Ring Lasers and Terrestrial Tests of Gravity. Galaxies, 3, 84-102. https://doi.org/10.3390/galaxies3020084

[3] Le Corre, S. (2015) Dark Matter, A New Proof of the Predictive Power of General Relativity. https://arxiv.org/abs/1503.07440

[4] Hobson, M., et al. (2006) General Relativity. Cambridge University Press, Cambridge. https://doi.org/10.1017/CBO9780511790904

[5] Mashhoon, B. (2008) Gravitoelectromagnetism: A Brief Review. https://arxiv.org/abs/gr-qc/0311030

[6] Clifford, M.W. (2014) The Confrontation between General Relativity and Experiment. Living Reviews in Relativity, 17, 4. https://doi.org/10.12942/lrr-2014-4 